\title{
Focal Plane Array IR imaging at the Australian Synchrotron
}

\author{
$\underline{\text { M. J. Tobin }}^{1}$, J. Vongsvivut ${ }^{1}$, D. E. Martin ${ }^{1}$, K. H. Sizeland ${ }^{1}$, M. J. Hackett ${ }^{2}$, R. Takechi ${ }^{2}$, \\ N. Fimorgnari ${ }^{2}$, V. Lam², J. C. Mamo², E. A. Carter ${ }^{3}$, B. Swarbrick ${ }^{3}$, P. A. Lay ${ }^{3}$, D. A. Christensen ${ }^{4}$, \\ D. Perez-Guaita ${ }^{4}$, E. Lowery ${ }^{4}$, P. Heraud ${ }^{4}$, B. R.Wood ${ }^{4}$, L. Puskar ${ }^{5}$, and K. R. Bambery ${ }^{1}$. \\ ${ }^{1}$ Australian Synchrotron, 800 Blackburn Road, Clayton, VIC 3168, Australia. \\ ${ }^{2}$ Curtin Health Innovation Research Institute, Curtin University, Bentley, WA 6102, Australia. \\ ${ }^{3}$ School of Chemistry, Vibrational Spectroscopy Core Facility, and Sydney Nano, \\ The University of Sydney, NSW 2006, Australia. \\ ${ }^{4}$ Centre for Biospectroscopy, Monash University, Clayton, VIC 3800, Australia. \\ ${ }^{5}$ Helmholtz-Zentrum für Materialien und Energie GmbH, Berlin, 12489 Germany.
}

\begin{abstract}
A Focal Plane Array FTIR microscope has successfully been coupled to the IRM beamline at the Australian synchrotron, following the method pioneered at previous beamlines at the SRC and NSLS I synchrotrons, whereby a wide aperture of synchrotron light is split into multiple beams and spatially reconfigured to match the entrance aperture of the FTIR instrumentation. Imaging performance has been assessed using a selection of polymer and biological samples, providing diffraction-limited sub-cellular lateral resolution in the biological materials. We have demonstrated that improved collection times at high lateral resolution are possible, when compared with single element point-mapping microspectroscopy, though this is achieved with a trade off in spectral noise. Future improvements in the use of an FPA detector at the Australian Synchrotron are proposed, including removal of coherent interference and installation of a dedicated beam extraction port for FPA microspectroscopy.
\end{abstract}

\section{Introduction}

The combination of IR microscopes with multi-pixel Focal Plane Array (FPA) detectors, high magnification optics and synchrotron sources showed great promise with the development of 
synchrotron infrared imaging beamlines at SRC (Madison WI) and NSLS I (Brookhaven NY), providing tissue-scale imaging at subcellular lateral resolution, with signal to noise not possible using benchtop FTIR instrumentation $[1,2]$. Since the closure of SRC and NSLS I, progress on IR imaging on third generation synchrotron sources with smaller photon extraction angles has shown that several challenges exist, including the need to spread an otherwise bright, well focused beam across an area detector and the inherent spatial structure of the defocused synchrotron beam [3,4]. Recent improvements in bench-top FTIR imaging instrumentation have shown that diffraction limited full field imaging with sub-wavelength pixel resolution can now be achieved through introduction of a magnifying optic between the objective and detector $[5,6]$. This, however, results in a reduction in photon flux per pixel, and for less strongly absorbing samples a Principal Component Analysis-based noise reduction algorithm has been employed to reduce spectral noise to acceptable levels [6]. The brightness and stability of a synchrotron beam, when combined with suitable coupling optics between the beamline and the FTIR microscope, enables high magnification IR imaging to be achieved with lower spectral noise than is otherwise possible.

We have followed the approach used at NSLS I [1], whereby the extracted fan of radiation from the Australian Synchrotron was split into four equal beams and re-configured into a $2 \times$ 2 "bundle". The necessary photon flux was achieved by combining the beam from the IR Microspectroscopy (IRM) and THz/Far-IR beamlines, providing a horizontal beam aperture of $58 \mathrm{mrad}$. The addition of piezo control of four focusing mirrors permitted in-vacuum refinement of the beam alignment through the Bruker V80v interferometer and Hyperion microscope. The remaining limitations of the IRM beamline at the Australian Synchrotron, when compared to previous beamlines at SRC and NSLS I [1,2], are the relatively low stored beam current of $200 \mathrm{~mA}$ (NSLS I current was 700 to $1000 \mathrm{~mA}$ ) and the maximum horizontal beam aperture of $58 \mathrm{mrad}$ (NSLS I aperture was $100 \mathrm{mrad}$, SRC aperture was $320 \mathrm{mrad}$ ). 
To demonstrate the performance of the IRM beamline operating with an FPA detector, selected samples were prepared and data were acquired during a dedicated user run of the beamline operating in this mode.

\section{Materials and methods}

The infrared beamline at the Australian Synchrotron extracts $58 \mathrm{mrad}$ horizontal and $18 \mathrm{mrad}$ vertical from the storage ring, using a slotted plane mirror inserted sideways into the dipole chamber [7]. The accepted fan of radiation extracted covers an angle from $-14 \mathrm{mrad}$ to +44 mrad either side of the centre line of the upstream straight section. The radiation extracted therefore includes both edge radiation from the entrance to the dipole magnet, and dipole radiation. The beam is refocused and directed downwards by a toroidal mirror, before being rotated and reflected horizontally by two plane mirrors. This provides a near $1: 1$ focus of the photon beam from the electron beam source to a point close to a wedged diamond window, separating the storage ring vacuum from the beamline vacuum. Downstream of the diamond window, under normal operating conditions, the expanding beam is separated horizontally during standard beamline operations, with approximately half of the beam being directed to the THz/Far-IR beamline and half being directed to the IRM beamline. For each beamline, the separated beam is refocused to a second focus point before being collimated with either an off-axis parabolic mirror (THz/Far-IR), or a near-normal incidence spherical mirror (IRM), with the cross section of the collimated beam matched to the FTIR instrument in use on each beamline.

\subsection{Beamline optics for FPA microspectroscopy}




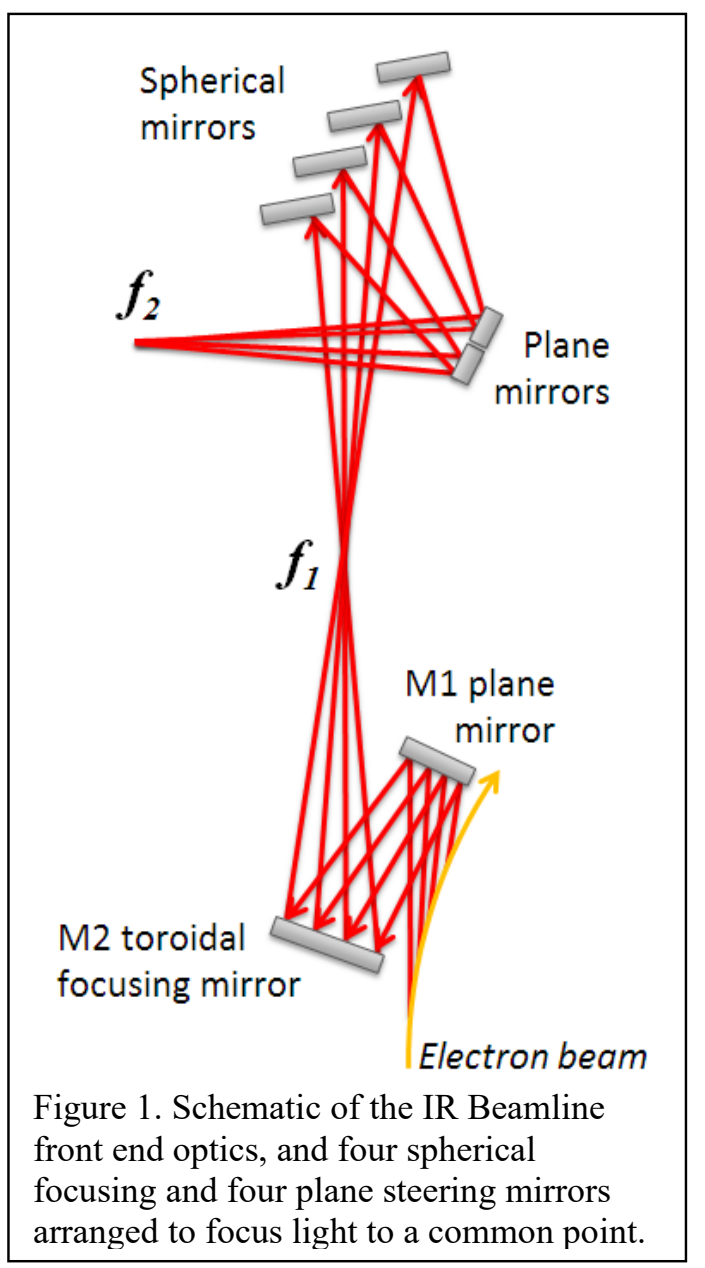

To enable infrared beam from the full $58 \mathrm{mrad}$ horizontal arc of the electron beam to be coupled to the Bruker V80v FTIR spectrometer and Hyperion 3000 microscope on the IRM beamline, a mirror system was installed that replaced the mirrors that usually separate the beam between the THz/Far-IR beamline and the IRM beamline. The new mirror system was designed with three main purposes: (1) to split the expanding beam horizontally into four equal components, which were each refocused to the same second focus position, therefore accommodating the extended depth of the electron beam source; (2) to reconfigure the refocused beam into a $2 \times 2$ "bundle" of beams that was more closely matched in dimensions to the circular entrance pupil of the Bruker Hyperion microscope optics; and (3) to enable fine adjustment under vacuum of the focusing mirrors included within this set up. Figure 1 shows a schematic of the beamline configured for four-beam FPA operation. The front end mirrors (M1 and M2) provide a beam focus f1 at the exit from the beamline diamond window. Downstream of focus $f_{1}$, the relative positioning of four spherical focusing mirrors and associated plane mirrors was such that synchrotron light emitted from four sections of the electron beam were refocused to a common focus $f_{2}$.

Figure 2A shows the four spherical focusing mirrors, with piezo controlled adjustment, each of which directed a portion of the synchrotron beam to one of the four plane mirrors that are shown in Figure 2B. The plane mirrors were independently adjustable such that the four 
reflected beams were brought to a common focus after exiting the vacuum chamber in which all eight mirrors were positioned. Figure $2 \mathrm{C}$ shows the relative position of the spherical and plane mirrors, with the direction of the four quarters of the synchrotron beam overlaid.
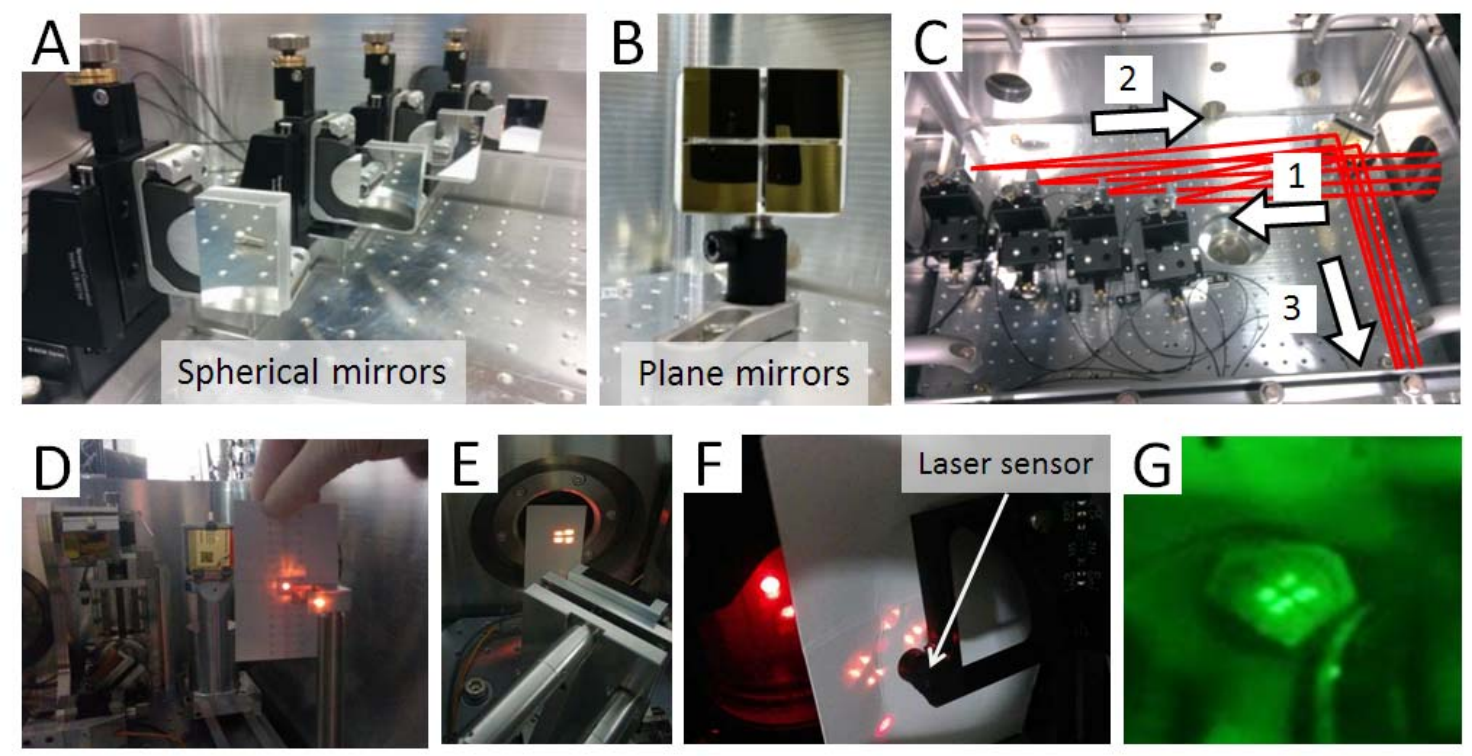

Figure 2. Alignment of the synchrotron beam for FPA imaging. (A) four spherical focusing mirrors, (B) four plane steering mirrors, (C) mirror arrangement and beam path to the spherical mirrors (1), the plane mirrors (2) and exiting the optics chamber to the second beam focus (3), (D) second beam focus, (E) four collimated beams entering the V80v spectrometer and $(\mathrm{F})$ passing the interferometer laser diode, and $(\mathrm{G})$ aligned onto the optical axis of the microscope objective.

\subsection{Alignment of synchrotron beam}

The four individually adjusted beams were brought to a common focus in the final optics chamber of the beamline (Figure 2D). The optics in this chamber were designed to produce a collimated beam from this focus point, which results in four parallel collimated beams exiting from the chamber (Figure 2E). The collimated beams then passed to the external source entrance port of the Bruker V80v and were aligned such that the four beams passed around the interferometer laser sensor diode (Figure 2F). The four beams then passed through the V80v interferometer and to the Hyperion 3000 IR microscope. An IRV2-2000 IR viewer (800 nm - $2000 \mathrm{~nm}$ range, Newport Corporation, Irvine, CA) was used to confirm that the 
four beams passed through the $20 \times$ condenser optic, and Figure $2 \mathrm{G}$ shows the four beams on an alignment target placed over the objective mounting thread, prior to installation of the objective.

\subsection{Infrared Microscope set up}

The Bruker Hyperion 3000 microscope was equipped with a mid-IR focal plane array detector with $64 \times 64$ active pixels. Optimum performance was achieved using the combination of a $20 \times(0.6$ Numerical Aperture) condenser (Bruker Optik GmbH, Germany) and a $52 \times(0.65$ Numerical Aperture) objective (Edmund Optics, Singapore), providing a detector pixel pitch of $0.77 \mu \mathrm{m} \times 0.77 \mu \mathrm{m}$ in the sample focal plane. Depending on the experimental requirement, the detector was operated either at the full frame size of $64 \times 64$ pixels, or with a cropped readout area of $32 \times 32$ or $16 \times 16$ pixels. Reading the reduced area ensured that spectra were collected only from the most evenly illuminated portion of the detector. The detector readout rates used were $5 \mathrm{kHz}$ for the FPA (actual integration time used $=0.2146 \mathrm{~ms}$ ) with a gain of 3 , and $40 \mathrm{kHz}$ for the single element MCT detector. The FPA detector had a long wavelength cut-off of $850 \mathrm{~cm}^{-1}$, whereas the single element MCT used had a cut-off of $750^{-1}$.

\subsection{Preparation of samples for FTIR imaging}

A selection of tests samples was prepared by participating research teams to enable the performance of the IRM Beamline with FPA detector to be assessed.

A polymer test pattern was prepared by using a chrome-on-quartz 1951 USAF resolution test pattern (Edmund Optics, Singapore) as a photolithographic mask to create a $2.5 \mu \mathrm{m}$ thick 
pattern replica with the photoresist SU8-2002 on $0.5 \mathrm{~mm}$ thick calcium fluoride windows (Melbourne Centre for Nanofabrication, Australia).

Mouse brain tissue sections, with early stages of amyloid aggregation, were mounted on 0.5 mm thick calcium fluoride windows.

Adipocytes (3T3-L1 murine cell line) were cultured onto calcium fluoride windows and fixed in cold methanol before washing in de-ionised water and air drying using similar procedures to those used for studies of adipocytes on silicon nitride windows [8-10], except calcium fluoride was used [11].

Live, hydrated Micrasterias hardyi unicellular algae were mounted between two $0.5 \mathrm{~mm}$ thick calcium fluoride windows, and the edge of the windows sealed with silicone grease. This organism is sufficiently robust to survive confinement in a liquid flow cell for many hours, and has been used as a model for the study of phytoplankton response to nutrient change in their environment $[12,13]$.

For all samples except the adipocytes, multi-FPA frame images were acquired using the Bruker OPUS v7.2 software, which controlled the stage movement to generate a mosaic image of the required number of FPA frames covering the region of interest. Data from adipocytes were collected as single frame $64 \times 64$ pixel FPA images.

\section{Results and Discussion}

\subsection{FTIR Imaging of test samples}

Alignment of the four beams through the Hyperion 3000 microscope resulted in a pattern of illumination that covered most of the area imaged by the $64 \times 64$ pixel area of the FPA detector, as shown in Figure 3A, with the central $32 \times 32$ pixels evenly illuminated. 
Adjustment of the piezo-controlled spherical mirrors shown in Figure 2B enabled the illumination of the central $32 \times 32$ pixels to be refined, as shown in Figures $3 \mathrm{~B}$ and $3 \mathrm{C}$. As indicated by the scale bar, the detector count per pixel ranged from 4000 counts to 8000 counts, with the maximum count rate approximately half of the maximum readout rate for the detector.
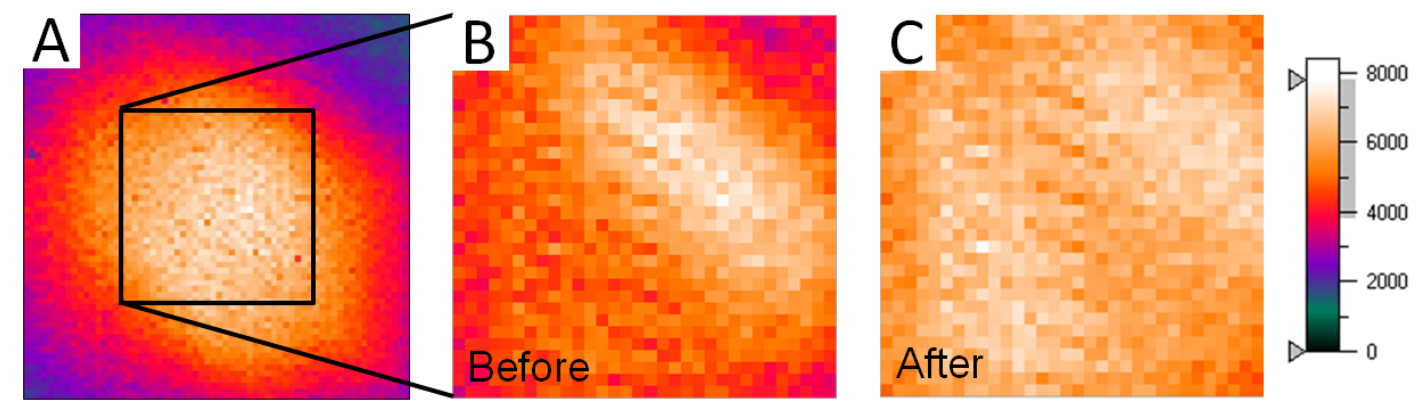

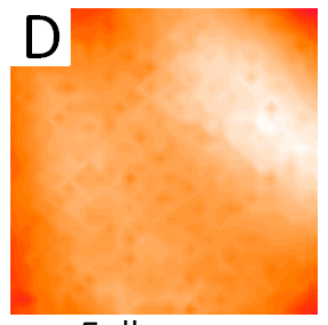

Full range $850-3850 \mathrm{~cm}^{-1}$

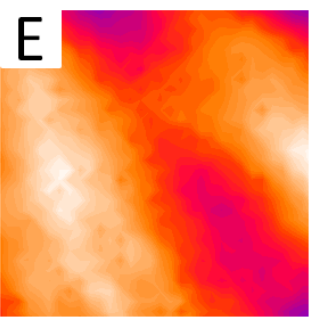

$2800-3000 \mathrm{~cm}^{-1}$

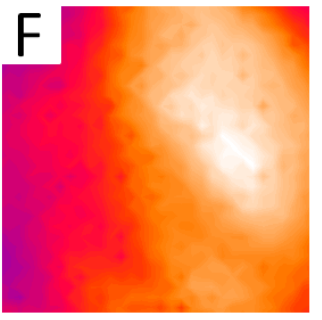

$1580-1720 \mathrm{~cm}^{-1}$

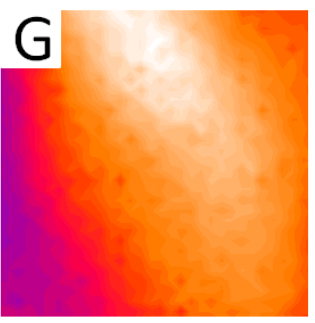

$1000-1150 \mathrm{~cm}^{-1}$

Figure 3. Infrared illumination of the FPA detector. (A) Full $64 \times 64$ pixel are of the array with central $32 \times 32$ pixels outlined, (B) central $32 \times 32$ pixels before adjustment of piezo-controlled mirrors and (C) after adjustment. Integrated intensity of infrared beam from single channel spectral data across (D) the full detected spectrum, (E) the $\mathrm{C}-\mathrm{H}$ stretch region, (F) the Amide I region, and (G) the symmetric phosphate stretch region.

The distribution of detector illumination in Figure 3C shows the direct readout from the FPA detector, and as such shows the total integrated intensity across the full spectral operating range of detection of the FPA detector. The distribution was however found to be strongly wavelength dependent, and this is shown in Figure 3D-G, where the intensity maps are derived from the reference single channel data acquired through the Bruker OPUS software, and integrated over the spectral ranges shown. 
Figure 4A shows to the left an absorption map of the SU8-2002 photoresist absorption peak between $1208 \mathrm{~cm}^{-1}$ and $1272 \mathrm{~cm}^{-1}$ comprising $6 \times 9$ FPA images, each of $16 \times 16$ pixels, covering an area of the USAF photoresist pattern bearing the number " 4 ". The width of the bars making up the number was $10 \mu \mathrm{m}$ and the edges of the bars showed a level of sharpness that would be expected at the diffraction limit for light of $8 \mu \mathrm{m}\left(1250 \mathrm{~cm}^{-1}\right)$. The resulting image shows good spatial matching between the edges of the individual tiles that make up the image. The variation in the apparent absorption was stronger at the intersection of the bars, which may reflect variation in the thickness of the photoresist following etching.

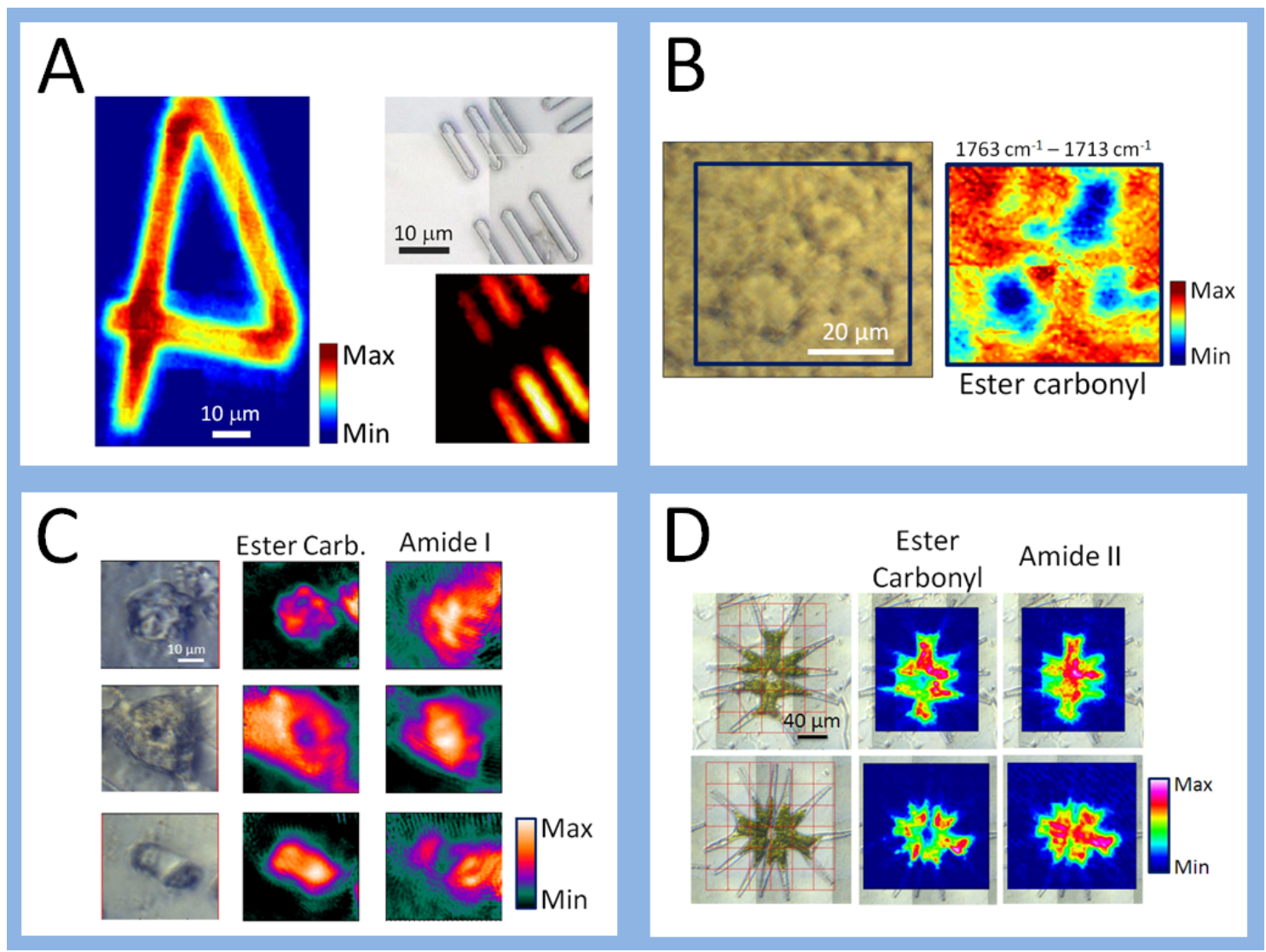

Figure 4. Example IR absorption images using the FPA detector. (A) photoresist test patterns on calcium fluoride, (B) microtomed mouse brain tissue- showing lipid distribution around three neuron cell bodies, (C) Lipid and Protein distribution in individual adipocytes, and (D) live, hydrated Micrasterias hardyi unicellular algae. 
Figure 4A also shows to the right a visible light image and corresponding IR absorbance map of a patterned photoresist in which the diagonal bars are $2.1 \mu \mathrm{m}$ wide, separated by $4.2 \mu \mathrm{m}$. Absorbance was integrated between $1474 \mathrm{~cm}^{-1}$ and $1525 \mathrm{~cm}^{-1}$.

The lipid distribution in the mouse brain tissue was analysed using single and multiple FPA frame maps. Each frame was collected at $8 \mathrm{~cm}^{-1}$ spectral resolution with 512 scans co-added. The example shown in Figure 4B illustrates the higher concentration of lipid surrounding three neuronal cell bodies that can be seen as roughly circular features with dark edges, 15-20 $\mu \mathrm{m}$ in diameter, in the visible light image to the left. The lipid distribution was shown by the integration of the ester carbonyl absorbance between $1763 \mathrm{~cm}^{-1}$ and $1713 \mathrm{~cm}^{-1}$, and is shown in a tiled image to the right that is comprised of 4 FPA frames of $32 \times 32$ pixels each.

Observation of a "halo" of high lipid around neuron cells in early stages of neurodegenerative disease has been reported previously [14].

Single cultured adipocytes were imaged as part of an ongoing study of anti-diabetic drugs [9]. Single cells, or pairs of cells were imaged using the full $64 \times 64$ frame of the FPA detector, without tiling multiple images. Examples of three cells are shown in Figure 4C, with visible light images to the left, and with lipid and protein maps to the right, produced by integration of ester carbonyl $\left(1713 \mathrm{~cm}^{-1}\right.$ to $\left.1763 \mathrm{~cm}^{-1}\right)$ and amide I $\left(1600 \mathrm{~cm}^{-1}\right.$ to $\left.1697 \mathrm{~cm}^{-1}\right)$ bands. Differences in the lipid and protein distribution can be seen. The intense amide I bands near the centre of the cells are due to the high concentrations of protein in the chromatin of the nucleus [8]. These regions correspond to the nucleus observed in the optical image, and as expected have low levels of lipid that are concentrated in the multiple lipid droplets that are external to the nucleus [8-11]. As also expected, the high concentrations of lipids correspond to the lipid droplets observed in the optical image. There are some interference patterns particularly observed in the amide image in the top right hand corner of Figure $4 \mathrm{C}$ and such 
patterns have been observed in FPA data from other IR beamlines (results not published) and may be a result of the near-coherence of the synchrotron source in the IR spectral range [15]. FTIR microspectroscopy of living, hydrated organisms is particularly challenging due to the strong absorbance by water of light in the mid-infrared. Previous published FPA-based studies of this organism have mainly been applied to air-dried samples, with one example of live cells $[12,16,17]$. Figure 4D shows two live examples of the unicellular microalga Micrasterias hardyi, which exists as a pair of "semicells". The IR maps of the upper and lower samples are comprised of $5 \times 6$, and $6 \times 6$ FPA frames, respectively, of $32 \times 32$ pixels each. Clear detail within each semicell was observed in the infrared maps of the ester carbonyl (lipid) and amide II (protein) absorbance bands, which broadly corresponded to the location and shape of the large chloroplast that was observed as the dark feature in the visible light images to the left. The ester carbonyl map more closely matched the chloroplast shape, while the amide II distribution extended to include the central region where the two semicells meet, and where the nucleus of the organism resides.

\subsection{Performance comparison with synchrotron point mapping.}

It is possible to make some comparisons between the performance of our synchrotron FTIR microscope operating with an FPA detector in imaging mode and operating with a single element MCT detector in point mapping mode, based on (a) the time taken to collect data from a given area of sample and (b) the percentage noise in the collected spectra. These comparisons are specific to the IRM beamline at the Australian Synchrotron. It should also be noted that the FPA detector has a relatively high cut-off wavelength of $850 \mathrm{~cm}^{-1}$, compared with $750 \mathrm{~cm}^{-1}$ for the narrowband MCT used here, and $450 \mathrm{~cm}^{-1}$ for wideband MCT detectors. 
(a) Speed comparison. The data from the mouse brain tissue and single adipocytes (Figure 4B and 4C) were acquired using 512 co-added scans, and 256 co-added scans respectively, at $8 \mathrm{~cm}^{-1}$ spectral resolution. Each image covers an area of $49 \times 49 \mu \mathrm{m}^{2}$ and was collected in approximately $13 \mathrm{~min}$ (mouse brain) and $6.5 \mathrm{~min}$ (adipocytes), with a pixel pitch of $0.77 \times$ $0.77 \mu \mathrm{m}^{2}$. To cover a similar area within $13 \mathrm{~min}$ by single point mapping at $8 \mathrm{~cm}^{-1}$ resolution would require the Bruker V80v FTIR spectrometer to accumulate no more than 8 scans $(\sim 3.5$ s per pixel), and for the Hyperion 3000 microscope to map the sample at a step size of $3 \mu \mathrm{m}$ in both $x$ and $y$ directions, to generate a map of just $16 \times 16$ pixels. If collecting fewer than 8 scans, the mapping method becomes limited by the microscope's stage movement and speed of starting each new scan, and this therefore represents the fastest possible speed in mapping mode. However, the multiplex nature of the FPA imaging method allows simultaneous acquisition from multiple pixel points, such that when fewer than 512 co-added scans are required, the data collection time for a given sample area by FPA is faster than is possible by the point mapping method.

(b) Noise comparison. To compare spectral noise, five spectra were selected from the centre of the mouse brain tissue map (512 scans), and the RMS noise was measured in the spectral region between $2450 \mathrm{~cm}^{-1}$ and $2550 \mathrm{~cm}^{-1}$ using the Bruker OPUS noise analysis routine, with a parabolic baseline fit. Spectra recorded from a similar tissue in point mapping mode with a single point MCT detector, $6 \times 6 \mu \mathrm{m}^{2}$ spatial aperture, 8 scans and $8 \mathrm{~cm}^{-1}$ resolution, were similarly analysed. Point mapped spectra showed an average noise of $0.038 \%$ RMS for 8 scans, while the FPA data showed average noise of $0.11 \%$ RMS for 512 scans. Pixel binning of the FPA data at $2 \times 2$ pixels (average of 4 pixels) reduced the average noise to $0.059 \%$ RMS, and binning data at $4 \times 4$ pixels (average of 16 pixels) further reduced noise to $0.043 \%$ RMS, approaching that of the point mapped data. At this point, each binned pixel 
corresponded to an area of sample of $3.1 \times 3.1 \mu \mathrm{m}^{2}$, approximately that of the step size required to map the same area in a similar time with a single element MCT detector.

These results indicate that for our FTIR microscope beamline, FPA imaging only achieved a similar noise level in IR absorbance spectra to single element mapping by collecting 64 times as many scans and averaging $4 \times 4$ pixels. Where fewer than 512 scans were required, for example where strong absorption peaks are being investigated, or where a higher pixel pitch may be required for improved lateral resolution, the FPA detector provided a faster data collection than was possible with single point IR mapping.

The relatively low signal to noise observed when operating in imaging mode with the FPA, when compared to single point mapping mode, may be due largely to the reduced number of photons delivered per pixel to the detector. At best, the beam intensity available through the four-beam configuration described here is only twice that routinely employed in standard synchrotron mapping acquisitions. Even if all of this light could be refocused onto the central $32 \times 32$ pixels of the FPA detector used in the measurements describe here, this still represents less than $1 / 500^{\text {th }}$ the intensity of light per pixel at the detector, when compared with point mapping with synchrotron light.

Potential improvements to the spectral noise and image quality with synchrotron FPA can be considered. Point mapping data at the Australian Synchrotron are collected using a gating system to reject scans collected during the synchrotron injection cycle, which happens every 3 to 5 min since the injection is known to perturb the beam and adds noise to FTIR data [18]. Implementation of a gating procedure for FPA collection is planned for future operations. Artefacts observed in many images, particularly at longer wavelengths, such as those present in Figure 4C (amide I), are thought to be due to coherent interference within the optical system. The use of a ground calcium fluoride beam diffuser, and refracting rather than 
reflecting optics have recently been shown to remove such effects in a laser-based FTIR microscope and may greatly improve performance with synchrotron light [15]. Finally, access to a higher flux of synchrotron light, such as was available at SRC and NSLS I $[1,2]$, will improve the advantage of FPA imaging over point mapping, since a single-element MCT detector is close to saturation even with the moderate IR flux available at the Australian Synchrotron. This may be advantageous at third generation synchrotrons operating with higher than $200 \mathrm{~mA}$ stored current and wider horizontal extraction angles than $58 \mathrm{mrad}$.

\section{Conclusions}

By following the method of coupling multiple synchrotron beams to a single FTIR instrument we have enhanced the performance of the IRM beamline at the Australian Synchrotron. Our results show that for this beamline, FPA Images, or mosaics of multiple FPA images collected with fewer than 512 co-added scans can be collected more quickly, and with equivalent or better lateral resolution than is possible with single point spectral mapping. There is however a trade off in that the spectral noise observed, when the synchrotron beam is distributed across a multi-pixel imaging detector, is higher than is observed in single point data accumulated over a similar timeframe. The ideal combination at a synchrotron IR beamline would be to have switchable optics optimised for illumination of either a single element MCT detector, or a multi-element FPA such that the ideal detector can be easily selected for the required experiment.

The small source size of the synchrotron electron beam, which is approaching he size of the infrared wavelengths being observed, results in a beam that is partially spatially coherent, and this can lead to imaging artefacts that are more commonly seen in laser-based imaging devices. Further exploitation of FPA detectors for synchrotron IR imaging is likely to require beam treatment to overcome this. 


\section{Acknowledgements}

The Authors are grateful to C.J. Hirschmugl (University of Wisconsin, Milwaukee, WI), M.J. Nasse (Karlsruhe Institute of Technology, Germany), L.M. Miller, G.L. Carr and R.J. Smith (Brookhaven National Laboratory, NY) for assistance and advice on the coupling of FPA FTIR microscopes to a synchrotron beamline. The data reported in this paper were collected at the Infrared Microspectroscopy Beamline at the Australian Synchrotron, which is part of ANSTO. The work was supported by the Australian Research Council (DP140100176 and DP160104172 to PAL), the Vibrational Spectroscopy Core Facility at the University of Sydney and the Australian Synchrotron. BS is grateful for support from a Henry Bertie and Florence Mabel Gritton Research Scholarship and an Australian Institute of Nanoscale Science and Technology (Sydney Nano, top-up scholarship).

\section{References}

1. Stavitski E, Smith RJ, Bourassa MW, Acerbo AS, Carr GL, Miller LM. Dynamic fullfield infrared imaging with multiple synchrotron beams. Anal Chem. 2013;85: 35993605. doi:10.1021/ac3033849

2. Nasse MJ, Walsh MJ, Mattson EC, Reininger R, Kajdacsy-Balla A, MacIas V, et al. High-resolution Fourier-transform infrared chemical imaging with multiple synchrotron beams. Nat Methods. 2011;8: 413-416. doi:10.1038/nmeth.1585

3. Hackett MJ, Caine S, Liu X, May TE, Borondics F. Development of single-beam wide-field infrared imaging to study sub-cellular neuron biochemistry. Vib Spectrosc. 2015;77: 51-59. doi:10.1016/j.vibspec.2014.12.004

4. Quaroni L, Zlateva T, Sarafimov B, Kreuzer HW, Wehbe K, Hegg EL, et al. Synchrotron based infrared imaging and spectroscopy via focal plane array on live 
fibroblasts in D2O enriched medium. Biophys Chem. 2014;189: 40-48.

doi:10.1016/j.bpc.2014.03.002

5. Findlay CR, Wiens R, Rak M, Sedlmair J, Hirschmugl CJ, Morrison J, et al. Rapid biodiagnostic ex vivo imaging at $1 \mu \mathrm{m}$ pixel resolution with thermal source FTIR FPA. Analyst. Royal Society of Chemistry; 2015;140: 2493-2503.

doi:10.1039/C4AN01982B

6. Hughes C, Henderson A, Kansiz M, Dorling KM, Jimenez-Hernandez M, Brown MD, et al. Enhanced FTIR bench-top imaging of single biological cells. Analyst. Royal Society of Chemistry; 2015;140: 2080-2085. doi:10.1039/C4AN02053G

7. Tobin M, Puskar L, Barber R, Harvey E, Heraud P. FTIR spectroscopy of single live cells in aqueous media by synchrotron IR microscopy using microfabricated sample holders. Vib Spectrosc. 2010;53: 34-38. doi:10.1016/j.vibspec.2010.02.005

8. Carter E a, Rayner BS, McLeod AI, Wu LE, Marshall CP, Levina A, et al. Silicon nitride as a versatile growth substrate for microspectroscopic imaging and mapping of individual cells. Mol Biosyst. 2010;6: 1316-22. doi:10.1039/c001499k

9. Wu LE, Levina A, Harris HH, Cai Z, Lai B, Vogt S, et al. Carcinogenic Chromium(VI) Compounds Formed by Intracellular Oxidation of Chromium(III) Dietary Supplements by Adipocytes. Angew Chemie - Int Ed. 2016;55: 1742-1745. doi:10.1002/anie.201509065

10. Bader CA, Carter EA, Safitri A, Simpson P V., Wright P, Stagni S, et al. Unprecedented staining of polar lipids by a luminescent rhenium complex revealed by FTIR microspectroscopy in adipocytes. Mol BioSyst. Royal Society of Chemistry; 2016;12: 2064-2068. doi:10.1039/C6MB00242K

11. Bader CA, Shandala T, Carter EA, Ivask A, Guinan T, Hickey SM, et al. A molecular 
probe for the detection of polar lipids in live cells. PLoS One. 2016;11: 1-24. doi:10.1371/journal.pone.0161557

12. Perez-Guaita D, Kochan K, Martin M, Andrew DW, Heraud P, Richards JS, et al. Multimodal vibrational imaging of cells. Vib Spectrosc. Elsevier B.V.; 2017;91: 4658. doi:10.1016/j.vibspec.2016.07.017

13. Heraud P, Wood BR, Tobin MJ, Beardall J, McNaughton D. Mapping of nutrientinduced biochemical changes in living algal cells using synchrotron infrared microspectroscopy. FEMS Microbiol Lett. 2005;249: 219-225. doi:10.1016/j.femsle.2005.06.021

14. Summers KL, Fimognari N, Hollings A, Kiernan M, Lam V, Tidy RJ, et al. A Multimodal Spectroscopic Imaging Method to Characterize the Metal and Macromolecular Content of Proteinaceous Aggregates (“Amyloid Plaques”). Biochemistry. 2017;56: 4107-4116. doi:10.1021/acs.biochem.7b00262

15. Serrano AL, Ghosh A, Ostrander JS, Zanni MT. Wide-field FTIR microscopy using mid-IR pulse shaping. Opt Express. 2015;23: 17815. doi:10.1364/OE.23.017815

16. Patel SA, Currie F, Thakker N, Goodacre R. Spatial metabolic fingerprinting using FTIR spectroscopy: investigating abiotic stresses on Micrasterias hardyi. Analyst. 2008;133: 1707. doi:10.1039/b809441a

17. Kansiz, Mustafa (Agilent Technologoes I., Rein, Alan (Agilent Technologoes I. Imaging of Live Cells in Water Using an Agilent 620 FTIR Microscope and an Agilent Cary 670 FTIR System Equipped with Standard Thermal Source. Agil Technol Tech Note. $2016 ; 1-4$.

18. Van Garderen ED, Bambery KR, Clift M, Leblanc GS, Martin DE, Puskar L, et al. Instrumentation upgrade for Top-Up operations at the Australian Synchrotron. J Phys 
Conf Ser. 2013;425. doi:10.1088/1742-6596/425/4/042015 